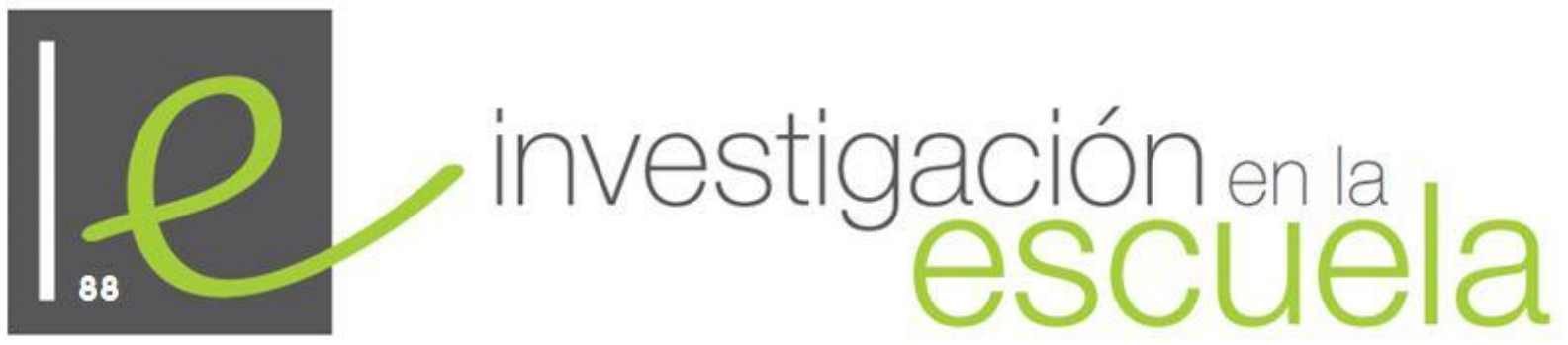

Revista académica evaluada por pares y de acceso abierto

\title{
Tercer espacio discursivo para aunar teoría y práctica en la enseñanza de las ciencias durante la formación en el Grado de Maestro en Educación Primaria
}

\author{
Ileana María Greca Dufranc \\ Universidad de Burgos \\ España
}

Citación: Greca, I.M (2016). Tercer espacio discursivo para aunar teoría y práctica en la enseñanza de las ciencias durante la formación en el Grado de Maestro en Educación Primaria . Investigación en la Escuela, 88, 63-77. Recuperado de: investigacionenelaescuela.es/articulos/R88/R88-4

Resumen: dentro del contexto de formación docente actual es necesario que los estudiantes de magisterio, además de una formación adecuada sobre las ciencias y sus metodologías didácticas, realicen una práctica reflexiva sobre su enseñanza, integrada en su práctica docente. Sin embargo, diversas investigaciones muestran que esta práctica reflexiva no es frecuente. En este trabajo se muestra como la creación de un tercer espacio discursivo puede permitir dicha reflexión. Este tercer espacio fue construido aunando el segundo período de prácticas de los alumnos y la realización del trabajo de fin de grado. Los resultados obtenidos a partir de un estudio de caso con tres estudiantes indican que esta estrategia fue fundamental para que los estudiantes comprendiesen mejor la metodología de la indagación y su uso en clase, permitiéndoles implementarla.

Palabras clave: "formación de maestros"; "enseñanza de las ciencias”: "indagación"; "práctica reflexiva"; "tercer espacio". 
Third discursive space for linking theory and practice in science teaching in the training of students of the Degree in Primary Education

Abstract: in the actual context of initial teacher education, it seems necessary that, together with an adequate training about science and its didactic methodologies, pre-service teachers have the opportunity to do a reflective practice on science education, integrating it into their teaching. However, different researches show that this frequently is not the case. The aim of this paper is to show how a third discursive space allows this reflective practice to take place. This third space was built combining the second period of practice and the degree final project. The results obtained using a case study indicate that this strategy was crucial for a better understanding of the methodology of inquiry and its use in the classroom. This understanding seems to have allowed the pre-service teachers to implement it successfully.

Key words: "pre-service teachers"; "science education"; "learning by inquiry"; "reflective practice"; "third space".

\section{Troisième espace discursif pour combiner la théorie et la pratique dans l'enseignement des sciences au cours de formation en éducation primaire}

Resumè: dans le cadre de la formation en cours, est nécessaire pour les futurs enseignants, plus une formation adéquate dans les sciences et leurs didactiques, de accomplir une pratique réflexive sur leur enseignement, intégré dans leur pratique pédagogique. Toutefois, plusieurs recherches montrent que cette pratique réflexive n'est pas commune. Cet article montre comment la création d'un troisième espace discursif peut permettre à cette pratique réflexive. Cette troisième espace a été construite en combinant la deuxième période de pratiques dans les écoles et le projet final du diplôme universitaire. Les résultats obtenus à partir d'une étude de cas avec trois étudiants indiquent que cette stratégie était essentielle pour améliorer la compréhension de la méthodologie d'enquête et l'utilisation en salle de classe. Cette compréhension a permis aux étudiants de mettre en œuvre avec succès.

Mosts clé: "futurs enseignants"; "enseignement des sciences"; "l'apprentissage par enquête"; "la pratique réflexive"; "troisième espace".

\section{Introducción}

El informe Nuttfield (Osborne \& Dillon, 2008) indica que para aumentar el escaso interés de los alumnos sobre temas científicos y tecnológicos es necesario cambiar la enseñanza de las ciencias en la escuela primaria, introduciendo las metodologías y estrategias propuestas por la investigación en el área de la didáctica de las ciencias. Cambio que, según este mismo informe, es necesario que comience en la propia formación de maestros. Pero enseñar ciencias en consonancia con las recomendaciones actuales implica una gran cantidad de desafíos para los maestros que se forman, como los relacionados con las decisiones sobre qué y cómo enseñar tópicos científicos específicos en ambientes que permitan aprender significativamente contenidos sobre ciencia y sobre la naturaleza de las ciencias. Para ello, necesitan tener amplios conocimientos y habilidades, incluyendo una comprensión profunda del contenido, el dominio de metodologías para promover tanto el aprendizaje significativo de los conceptos como el envolvimiento de los alumnos en prácticas científicas y un conocimiento de estrategias para evaluar y monitorear los aprendizajes. Además, es necesario que sepan cómo manejar el comportamiento del día a día y tengan un repertorio de actividades para otras asignaturas durante todo el año.

Para alcanzar este conjunto de conocimientos y habilidades el informe Nuttfield, después de analizar diversas experiencias de formación de maestros en Europa, aboga por un modelo en el que 
exista un balance entre los componentes teóricos (conocimiento sobre las ciencias y sobre la enseñanza de las ciencias) y los prácticos (amplio conjunto de conocimientos tácitos que solamente pueden ser adquiridos a partir de la práctica en el aula). Sin embargo, los cursos de formación de maestros en muchos países, entre ellos España, enfatizan excesivamente los componentes teóricos de la formación, y los estudiantes, luego de sus períodos de prácticas, terminan con la visión que las teorías o métodos didácticos son irrelevantes para la práctica real de sala de aula (Cortés et al. 2012; Korthagen et al., 2006). De hecho, consideran que los que saben lo que es necesario enseñar y cómo hacerlo son sus mentores de prácticas de los centros escolares quienes, a su vez, utilizan como actividades predominantes en ciencias las relacionadas con el uso del libro de texto, la metodología expositiva (Cañal et al., 2013) y la evaluación mediante exámenes. Además, por regla general, los contextos de prácticas ofrecen escasas posibilidades a los estudiantes para implementar metodologías innovadoras sobre la enseñanza de las ciencias (Sykes \& Bird, 1992; Crawford, 1999).

Si tenemos en cuenta que nuestros estudiantes de magisterio no han tenido posibilidades de aprender ciencias de una forma que no sea la tradicional, que la tendencia es a enseñar tal como fueron enseñados (Britzman, 1986) y que no tienen oportunidades de poner en práctica metodologías innovadoras para la enseñanza de las ciencias, nos enfrentamos a un círculo vicioso del que resulta difícil poder salir. La literatura indica que es necesario que los estudiantes de magisterio además de una formación adecuada sobre las ciencias y metodologías didácticas, realicen una práctica reflexiva sobre su enseñanza, integrándolas dentro de toda su práctica docente. Como indican Bryan \& Abell (1999) es necesario que los formadores de maestros "entrenen" a sus estudiantes para que sean capaces de indagar de forma intencionada y sistemática sobre su propia práctica, brindando oportunidades para que reflexionen y aprendan de esas experiencias.

Dado el escaso tiempo disponible en las carreras de magisterio (Schwarz, 2009) es necesario encontrar formas efectivas de ayudar a los estudiantes para que puedan enseñar ciencias de forma exitosa. En este sentido, dentro de la estructuración del Grado en Maestro de Educación Primaria (RD 861/2010, de 3 de julio), parece viable la construcción de un "tercer espacio discursivo", aunando el segundo período de prácticas de los estudiantes y la realización del trabajo de fin de grado.

El tercer espacio, un concepto tomado de los estudios poscoloniales (Moje et al., 2004), es entendido como un espacio discursivo híbrido en el que se encuentran discursos alternativos, competitivos entre sí, donde es posible transformar el conflicto y las diferencias en zonas de colaboración y aprendizaje (Gutiérrez, Baquedano-López \& Turner, 1997). Gutiérrez et al. (1999) señalan que esta noción puede ser productiva para comprender las complejidades de los ambientes de aprendizaje y su potencial transformador, pudiendo ser entendido, desde la teoría de la actividad, como una actividad expandida (Engeström, 1999), donde el objeto de la actividad es extendido y la actividad en sí reorganizada, resultando en nuevas oportunidades de aprendizaje. Así, estas zonas de actividad expandidas operan como zonas de desarrollo proximal. En nuestro caso, la actividad, que corresponde a la práctica de los estudiantes, se extiende para adicionar una reflexión crítica de esa propia práctica, fundamentada en el conocimiento teórico adquirido durante la carrera, proporcionándoles oportunidades para desarrollar nuevos aprendizajes. Este tercer espacio funde el "primer espacio" emergente del entorno cotidiano de los sujetos y que determina en gran medida sus concepciones personales, con el "segundo espacio" que encuentran en ambientes más formales, como la escuela. Aunque en general la identificación de primer o segundo espacio es arbitraria, dependiendo de la visión del investigador en relación a los diferentes discursos que se encuentran en el tercer espacio, la idea es que uno de ellos es dominante o privilegiado en la interacción social y el otro es marginalizado. Lo importante es que estos espacios pueden ser reconstruidos para formar un tercer espacio discursivo, del que emerjan nuevos conocimientos. En el caso que se presenta en este trabajo, los discursos conflictivos que se encuentran en el tercer espacio son los determinados por el 
conocimiento teórico brindado por la formación universitaria (específicamente, en nuestro caso, el conocimiento acerca de la enseñanza de las ciencias) y el conocimiento práctico formado por las concepciones de los maestros en formación acerca de las ciencias y su enseñanza, generadas por sus vivencias como alumnos, y lo que observan durante sus prácticas. Este espacio híbrido puede permitir un diálogo fructífero y valioso entre los maestros en formación y los formadores de maestros de la universidad acerca de los problemas de la práctica relacionados con la enseñanza de las ciencias, ayudando a reconsiderar y desarrollar comprensiones más profundas de los problemas emergentes de la práctica y donde puedan ser propuestas y exploradas soluciones alternativas a dichos problemas. En este sentido, este tercer espacio permite trabajar con los docentes en formación de forma similar a lo que propone el proyecto IRES (Porlán y Rivero, 1998; García Pérez, 2000) para los docentes en activo.

Operacionalmente, la construcción del tercer espacio puede establecerse mediante la realización, durante el trabajo de fin de grado (TFG), de una unidad didáctica innovadora sobre contenidos de ciencias, que los estudiantes desarrollan e implementan en su período de prácticas y cuyos resultados o efectos deben analizar. Aunque, en principio, el período de prácticas debería permitir a los estudiantes reflexionar sobre lo que observan y sobre las clases que dan, la exigencia que investiguen de forma intencionada posibilita una reflexión más fundamentada y crítica de esa práctica.

Siendo un proyecto del área de didáctica de las ciencias experimentales de la Universidad de Burgos formar maestros que sean capaces de introducir la metodología de indagación en la escuela primaria, las secuencias elaboradas privilegian esta metodología. Cuando se habla de indagación en enseñanza de las ciencias (NRC, 2000; 2012; Cortés et al. 2012; Minner et al., 2010) se hace referencia a las actividades de los alumnos que les permitan desarrollar tanto el conocimiento y la comprensión de las ideas científicas como el entendimiento de la forma en que los científicos estudian el mundo natural (National Science Education Standars, 1996). En este sentido, la indagación implica un proceso intencional de diagnóstico de problemas, crítica de experimentos y distinción de alternativas, planificación de investigaciones, investigación de conjeturas, búsqueda de información, construcción de modelos, debate con compañeros y construcción de argumentos coherentes.

En este trabajo se describe la construcción de ese tercer espacio así como algunos temas que emergieron en él y que posibilitaron que los estudiantes mejorasen su comprensión sobre la aplicación de la metodología de indagación en sala de aula.

\section{Diseño de investigación}

Diversos informes internacionales abogan hoy por una enseñanza de las ciencias mediante la indagación científica (NRC, 2010, 2012; Rocard, 2007). Sin embargo, los resultados de la investigación (Cañal, 2000; Murphy et al., 2007; Porlán et al., 2010) muestran que, en general, los maestros tienen un conocimiento muy pobre de y sobre las ciencias, siendo muy difícil que puedan implementar estrategias por indagación, metodología que, además, no han vivenciado en su formación anterior (Haefner \& Zemba- Saul, 2004, Windschitl, 2002). Por ello, en las asignaturas de ciencias del grado de nuestra facultad hemos partido del supuesto que si los estudiantes del grado pueden experimentar de forma positiva indagaciones en su aprendizaje es más probable que las repliquen en su docencia. Esta propuesta (Greca et al., en prensa) consta de diversas asignaturas, en la última de las cuales, realizada durante el primer semestre del cuarto curso, antes del segundo período de prácticas, los estudiantes llevan a cabo indagaciones sobre un tema y luego desarrollan una unidad didáctica basada en dicha metodología. Los resultados obtenidos con la primera 
implementación de la propuesta muestran que la mayor parte de los estudiantes pasan a considerar la viabilidad y la necesidad de utilizar la metodología de indagación para la enseñanza de las ciencias en primaria como forma de superar formas tradicionales y no efectivas de enseñarlas. De ese grupo de estudiantes $^{1}$ (con ingreso en el grado en 2010-2011), 8 de ellos eligieron realizar el TFG en el área de la didáctica de las ciencias experimentales. Con 3 de estos alumnos fue posible construir ese tercer espacio, aunando, con el mismo tutor de la universidad (la autora de este trabajo), el TFG y las prácticas.

Estos alumnos fueron asignados en diciembre y se habló con los mentores de los centros escolares acerca de la viabilidad de implementar, durante las prácticas, una secuencia didáctica basada en la indagación sobre algún contenido científico de los previstos para ese período. En principio acordaron que sería posible, a pesar de mostrarse escépticos, con una reacción semejante a la descripta por Windschitl (2002): los maestros creen que enseñar ciencias por indagación es tedioso, exige demasiado trabajo y sólo es apto para alumnos por encima de la media, no pudiéndose integrar en las clases habituales. También es necesario destacar que ninguno de los tres mentores era responsable de dar la asignatura de conocimiento del medio a sus alumnos, a pesar de lo cual o bien indicaron la viabilidad de hacerlo en algunas de sus horas (como las de tutoría y plástica) o bien hablaron con sus colegas responsables de esa asignatura para desarrollarlo en sus clases.

En febrero de 2014, los estudiantes comenzaron su período de prácticas. Dado que estuvieron en escuelas de tres localidades diferentes, se optó por realizar pocas reuniones presenciales, pero con una intensa comunicación (por e-mail y telefónica).

Para garantizar el espacio de reflexión se decidió utilizar, para acompañar la práctica, el modelo de práctica reflexiva de Barnett (1992), que propone abordar casos reales del escenario profesional de cada uno de los participantes con todos los elementos contextuales que permitan mejorar la formación práctica. Los estudiantes debían escoger 4 casos reales, -- observados durante su estancia en el centro y en los que podrían o no haber tenido algún tipo de intervención -reconstruirlos, contrastarlos con su formación teórica, intentando cuestionar las creencias y teorías implícitas que podían observar en el caso, reflexionando sobre la influencia de este caso para su formación y las posibles lagunas y/o conflictos formativos detectados. Esta reflexión era comentada por escrito por sus compañeros. Luego de esa puesta en común, el estudiante hacía una reflexión final, proponiendo, si fuera el caso, posibles mejoras. Para ello se creó una carpeta on-line en las que los alumnos colgaban sus casos y sus compañeros los comentaban, discutiéndose luego en las reuniones presenciales. Se formó así una pequeña comunidad de práctica. Estos casos, con las reflexiones individuales y grupales, constituyeron una parte importante de la memoria de prácticas de los alumnos.

Por otra parte, una vez que se tuvo conocimiento del tema de ciencias a ser abordado, los estudiantes comenzaron a diseñar las unidades didácticas por indagación. La construcción de estas unidades implicó una fuerte interrelación y discusión con el profesor tutor de la universidad para poder planificarlas. En algunas secciones de estas unidades los estudiantes adaptaron material diseñado por ellos o por sus compañeros en las asignaturas del área que cursaron en la universidad.

Posteriormente, se discutió con cada estudiante los aspectos que les interesaría investigar acerca de la implementación de sus unidades, estableciéndose, por consenso, las preguntas de investigación, el tipo de metodología y las fuentes de datos.

A su vez, durante la implementación de las unidades, fueron observados por la tutora de la universidad al menos una vez, discutiéndose posibles mejoras después de esta observación. Además,

\footnotetext{
${ }^{1}$ Con todo los estudiantes de este grupo, alrededor de 130 alumnos, se llevó a cabo la investigación que aparece en Greca et al. (en prensa). Cabe destacar que la mayoría de ellos había seguido bachillerato de letras y sentían un particular rechazo hacia las ciencias, como relatado en dicho trabajo.
} 
se pidió a los mentores de los centros que comentasen con los estudiantes estas clases, sobre todo teniendo en cuenta su conocimiento acerca de los niños con los cuales se trabajaba. El trabajo de fin de grado incluyó, junto con el diseño de la unidad y el análisis de los resultados obtenidos, una reflexión global sobre su impresión del desarrollo de las unidades a partir de la evidencia obtenida, en vistas al conocimiento profesional adquirido.

En este contexto se decidió estudiar en qué medida el espacio de reflexión construido era útil para auxiliar a los estudiantes de magisterio no solamente a dominar mejor la metodología de indagación y a sentirse con confianza para usarla, sino también a integrar esta metodología junto con aspectos generales de la conducción del grupo (conducta, evaluación, etc.) que son muchas veces problemas que se suman a sus dificultades en ciencias.

Este estudio usa un método de caso múltiple diseñado para determinar las semejanzas y diferencias entre los alumnos (Creswell, 1998). El objetivo fue explorar en profundidad como estos estudiantes van desarrollando su comprensión sobre la utilización de la indagación científica dentro del contexto general de la actividad del aula y en qué medida las reflexiones fruto de su práctica y de su propia investigación acerca de esa implementación, les permite ir adquiriendo un conocimiento que eventualmente les puede ser útil para implementarla en clase. Fueron usadas varias fuentes de datos: los informes escritos realizados por los estudiantes (memoria de prácticas y memoria del TFG); las observaciones de las clases; el cuaderno de campo de las reuniones del tutor de la universidad (al final de cada reunión se anotaba lo abordado y las cuestiones y respuestas de los alumnos) y un cuestionario final, pasado un mes después de graduarse, en el que se les pidió su visión acerca de la experiencia vivida y la enseñanza de las Ciencias en comparación con sus ideas anteriores. Además de este material, específico del período de prácticas, se contó con sus respuestas a otros cuestionarios así como las unidades didácticas que habían diseñado en la asignatura del primer cuatrimestre. A medida que se realizaban los encuentros con los estudiantes, se fue leyendo repetidamente el material empírico recogido, para desarrollar un contacto profundo, intentando buscar temas que indicasen reflexiones relacionadas con la enseñanza de las ciencias, la indagación y su aplicación en el aula, que posiblemente no pudieran haberse realizado sin la existencia del tercer espacio. El análisis temático fue escogido para este proceso dada su flexibilidad y la posibilidad de determinar, sin una rígida idea anterior, patrones en las reflexiones de los estudiantes (Brown \& Clarke, 2006). De estos temas ${ }^{2}$, se escogieron aquellos que aparecían de forma recurrente, tanto en los encuentros presenciales como en el material escrito, desarrollándose categorías de análisis, que fueron luego aplicadas al material completo de los tres estudiantes.

\section{Resultados}

Como se ha indicado, en el tercer espacio discursivo entran en conflicto los saberes prácticos y teóricos de los estudiantes. Por ello, en la primera parte de esta sección, se discute su formación anterior al grado, sus ideas, expectativas y motivaciones en relación a las ciencias y su enseñanza y su visión de la práctica docente, elementos centrales del saber práctico que poseen, así como su desempeño en una asignatura del grado en la que fue usada la indagación como metodología. Para ello, son utilizados diversos documentos, en particular sus respuestas a algunos cuestionarios y sus diseños de unidades didácticas producidos en los años anteriores al segundo período de prácticas.

\footnotetext{
${ }^{2}$ Los temas iniciales incluían: la relación tanto con el centro escolar como con el mentor; la forma de trabajo de la clase en conocimiento del medio; el uso del libro de texto; las dificultades en ciencias de los alumnos; la conducta, diversidad y forma de trabajo en las clases; las ideas previas, su detección y formas de superación; la organización de la secuencia didáctica; las posibilidades de realizar
} 
Pablo, Ana e Isabel iniciaron el Grado con formaciones diferentes. Pablo había cursado el bachillerato Tecnológico y demostró, desde la primera asignatura de ciencias del Grado, un marcado interés hacia las mismas, en particular la Física, y era partidario de enseñar las ciencias de "forma práctica". Ana e Isabel venían de un bachillerato de Humanidades. Al iniciar el grado, manifestaban un rechazo hacia las ciencias y consideraban al libro de texto como el principal recurso para enseñarlas. Como indica Ana:

"Había optado siempre por la rama de las letras y nunca me había interesado por nada de carácter científico. De hecho, tenía total repulsión por las matemáticas, la física y la química. Por aquel entonces, pensaba que el libro de ciencias era útil para la enseñanza, por mis escasos conocimientos. No me veía capacitada para enseñar ciencias, la verdad, pero sí creía que era importante para los alumnos de primaria. El problema era que esos niños se encontrasen con profesores que odiasen las ciencias, se sintieran inseguros y tuvieran que enseñar ciencias por obligación" (fuente: cuestionario final).

Sin embargo ambas, después de cursar las asignaturas de Ciencias en el grado, querían probar si era posible enseñar ciencias a los niños de manera que les generase interés.

Ninguno de ellos había tenido experiencia durante su formación anterior al ingreso al grado con la metodología de indagación, aunque Pablo había realizado los característicos trabajos prácticos cerrados de Física en secundaria. En la disciplina de cuarto curso, en la que desarrollaron indagaciones a partir de problemas abiertos, tal como planteado en la introducción de este trabajo, los tres se desenvolvieron bastante bien, comprendiendo y usando de forma efectiva la emisión de hipótesis, el diseño de experimentos y la construcción de modelos y argumentos para dar respuesta a las preguntas a partir de la evidencia empírica. Sin embargo, no todos consiguieron diseñar unidades didácticas aplicando correctamente la metodología de indagación. Isabel partió de una situación problemática bien planteada y su unidad estaba diseñada de forma adecuada para conseguir que los niños respondiesen a esa situación siguiendo todo el proceso de la indagación. Ana, sin embargo, planteó una situación confusa y una serie de procedimientos experimentales no claramente secuenciados, siendo poco probable que los alumnos consiguiesen resolver el problema y aprender los conceptos científicos que proponía. Pablo, a su vez, no consiguió diseñar una secuencia que usase efectivamente la indagación. Si bien partió de un problema potencialmente interesante, la secuencia de actividades propuesta no conseguía dar respuesta al problema y quedaba más como actividad "práctica", de hacer con las manos, sin que propusiese actividades en las que explícitamente se permitiese a los alumnos construir algún conocimiento científico.

Por otra parte, los tres han sido estudiantes muy comprometidos con la enseñanza, habiendo elegido la carrera por vocación y que habían realizado un primer período de prácticas valorado como excelente por sus tutores de la universidad y de los centros escolares. Cabe destacar que a la vuelta del primer período de prácticas, gran parte de los estudiantes de toda la promoción se sentía, en general, poco a gusto con la formación que estaban recibiendo, clasificándola de muy teórica. Pablo, en particular, acusó esta insatisfacción de forma muy marcada.

"También me quiero reiterar sobre el hecho de que el objetivo de estas prácticas es arrojar luz y dotar de un sentido a todo lo que se trabaja; hacer actividades teniendo en cuenta la conducta de los alumnos, los tiempos reales de la clase, asumir que estás solo para dar cabida a las necesidades educativas especiales de tus alumnos y, en resumen, partirte la cabeza para que todo cuadre, pero hacerlo. Es por esto que opino que estas prácticas son reveladoras para cualquier alumno que desee sacar un mínimo provecho de esta carrera, y abogo por adelantar este practicum al segundo año del grado, con el fin de no perder el tiempo que otros hemos perdido sin pensar o mejor dicho sin saber lo que es un niño, cómo se comporta y qué cosas le motivan de verdad.” (fuente: Memoria de prácticas) 
A continuación, se presentan algunos de los temas recurrentes que aparecen en los tres estudiantes. Por cuestión de espacio, serán ejemplificados con el caso de Pablo, que fue escogido porque, a pesar de tener alguna formación científica, fue el que más dificultades tuvo para poder diseñar e implementar su secuencia didáctica.

\section{La conducta de clase}

Aprender a gestionar la conducta de la clase es una preocupación central en todos los estudiantes de prácticas. De hecho, sienten que los maestros que lo consiguen, son los que saben y sus técnicas, las únicas posibles. En el caso de Pablo, su mentora del centro conseguía mantener un clima razonable de trabajo, aún con un par de alumnos que generaban desorden. Una particularidad de la metodología de la indagación es el trabajo grupal; sin embargo no suele ser utilizado normalmente en las aulas, como en el curso en que Pablo realizaba sus prácticas. Así, a pesar de haber aprendido las ventajas "teóricas" (como él decía) del trabajo grupal, era reticente a utilizarlo, alegando que los niños no estaban acostumbrados a trabajar de esa forma y que podía ser complicado. Luego de varias discusiones sobre el diseño de la unidad por indagación, aceptó probar si era posible. Para su sorpresa los niños trabajaron en general bien y los problemas que surgieron estuvieron relacionados con su propia inexperiencia para combinar (para la edad de 7 años) autonomía y dirección.

"Sin embargo, hubo también carencias a nivel del profesorado, cuya responsabilidad asumo totalmente, puesto que el orden de clase que debería haber impuesto siendo la primera vez que se trabajaba con esta metodología no lo impuse; esto pudo contribuir a la susodicha pérdida de tiempo" (fuente: Memoria del TFG).

En este caso, la reflexión en el tercer espacio, fruto de la interacción entre la teoría y la aplicación de la misma en un contexto real, ayudó a Pablo a convencerse de la efectividad del trabajo grupal. Esto le permitió, por una parte, implementar de hecho la metodología de indagación pues su secuencia didáctica, sin el trabajo en grupo, no hubiese sido una indagación. Y, por otra, le permitió darse cuenta que los posibles problemas que pueden aparecer con el uso de esta forma de organización están más relacionados con la capacidad del maestro de gestionarlos que con la inmadurez de los alumnos o utopías teóricas.

\section{El trabajo con niños con necesidad educativas especiales}

En su formación, Pablo tuvo asignaturas relacionadas con la educación especial y, aunque no era su especialidad, su vivencia del Practicum I con el mismo grupo de alumnos, lo había sensibilizado hacia la problemática del trabajo con estos alumnos:

"Por último, y como ya hiciera en mi reflexión el año pasado, me gustaría hacer mención a la atención a la diversidad. Siento como imposible que un solo maestro pueda atender debidamente a un alumno con problemas severos o retrasos madurativos importantes él solo. Simplemente es imposible" (fuente: Memoria del Practicum).

Con Pablo se discutió sobre si era posible introducir la indagación en una clase normal, con alumnos con distintas capacidades cognitivas. De allí surgió una de sus preguntas de investigación acerca de que si había diferencias entre los resultados conseguidos, en términos de aprendizaje, por los alumnos usando la metodología tradicional y la indagación. En particular, se quería investigar si esta metodología ayudaba a aprender ciencias a niños con dificultades de aprendizaje. Sus propios resultados le mostraron que no solamente los alumnos con altas capacidades consiguieron beneficiarse del uso de esta metodología, sino que también era posible y beneficioso para aquellos con menores capacidades cognitivas. 
"Como conclusión, podríamos decir que este tipo de unidad y de evaluación parece ayudar especialmente a chicos con problemas para la abstracción, ya que la ciencia se refiere a todo lo que nos rodea y puede ser representable para estos niveles, favoreciendo de esta forma sus comprensiones iniciales sobre los conceptos. Los alumnos brillantes son también los principales beneficiados de este tipo de metodología: mejoran su interacción social con el trabajo en grupo, vivencian contenidos que de otro modo probablemente sólo estudiarían de manera abstracta y inconexa del mundo en el que viven y aprenden de manera significativa, sentando bases científicas para futuros procederes" (fuente: Memoria del TFG)

En este tema se observa como el tercer espacio permitió a Pablo una nueva comprensión tanto de la metodología de la indagación como del trabajo con niños con necesidades educativas especiales. Cabe destacar que las dudas de Pablo solamente se vieron disipadas cuando investigó su propia acción docente, a partir del análisis de los resultados obtenidos por los alumnos en diferentes actividades(independientemente de la motivación con la indagación que consiguió con todos los alumnos) y resultó un descubrimiento que parece haber marcado su visión de la enseñanza de las ciencias de esta manera.

\section{Las dificultades para enseñar ciencias por indagación}

Como se ha indicado, Pablo era un entusiasta de la enseñanza de las ciencias de forma "práctica" y tenía una base razonablemente buena sobre el tema que debía realizar la secuencia didáctica (fuerzas). Sus ideas iniciales podrían caracterizarse dentro de una enseñanza "tradicional activa", una concepción de transición entre una perspectiva tradicional y una constructivista:

"Pensaba mostrar a los alumnos una serie de experiencias relacionadas con las fuerzas y a partir de allí, explicarles. Esto, a su vez, era coherente con su visión personal: "Sobre las ciencias pensaba que siempre nos explicaban menos de lo que yo quería saber cuando iba al colegio" (fuente: Cuestionario final).

Además, era también coherente con su visión de la gestión de la conducta de clase, como se discutió anteriormente. Así, a pesar de tener, en principio, herramientas adquiridas en asignaturas anteriores para poder diseñar secuencias por indagación, su historia personal (a la que hay que agregar sus preocupaciones sobre el trabajo en el aula, discutidas antes) y la práctica escolar parecían impedirle diseñar algo más acorde con la metodología. Fue necesario reflexionar con Pablo detenidamente los fundamentos de la metodología de la indagación, reduciendo los conceptos que iba a trabajar y relacionándolos con las ideas previas de los niños. Finalmente consiguió diseñar una buena secuencia acerca de su tema, compatibilizando tanto los aspectos teóricos de la metodología como las propias limitaciones de la práctica escolar. La secuencia quedó configurada a partir de un problema y estaciones de trabajo en la que los alumnos experimentaban por sí propios, emitían hipótesis, las experimentaban y verificaban si coincidían o no con sus ideas iniciales. Luego de pasar por todas las estaciones, el maestro retomaba las preguntas de las estaciones e incentivaba una discusión sobre aspectos conceptuales partiendo de los resultados obtenidos por los alumnos para conseguir mejorar los modelos iniciales.

En este punto se puede observar cómo, a pesar de los esfuerzos del cuerpo docente del área de didáctica de las ciencias de trabajar sistemáticamente con la metodología de indagación con los estudiantes y del hecho de que Pablo no tenía los impedimentos que la literatura denota como los más importantes para no implementarla en el aula (falta de interés por la ciencia, escaso conocimiento científico, ideas muy ingenuas sobre la naturaleza de las ciencias), no consiguió por si sólo diseñarla. Y parte de sus dificultades para este diseño estuvieron relacionadas con su propia visión de la enseñanza de las ciencias y la forma en que entendía la práctica escolar. Solamente la reflexión sobre la aplicación en el aula, en un caso concreto, permitió que empezase a comprender 
las características centrales de la metodología y su forma de aplicación, superando sus propias ideas y preconceptos iniciales sobre la enseñanza de las ciencias y convenciéndose efectivamente de sus ventajas. Esto puede verificarse contrastando, de una parte, su visión previa del uso de la indagación, según un cuestionario que contestó al finalizar la disciplina del grado en la que trabajaron con indagaciones:

"Si a mi criterio, soy capaz de realizar una unidad que me permita usar la indagación, la usaré, pero si por el contrario no encuentro una forma que cumpla con las necesidades formativas de mis alumnos, trabajaría de otra forma, como por ejemplo, incluyendo experiencias de laboratorio para afianzar los conocimientos" (fuente: Cuestionario al finalizar la asignatura, noviembre 2013)

Y de otra, su reflexión posterior al proceso de implementación y reflexión sobre su propia práctica:

"Para empezar descubrí la poca idea que tenía sobre la enseñanza de las ciencias, y eso fue todo un cambio. Abrí la mente a nuevas metodologías y fue un gran acierto. Hay formas de enseñar ciencias que dan grandísimos resultados, encandilan a los niños y no suponen tanto esfuerzo por parte del maestro" (fuente: Cuestionario final, agosto 2014).

\section{La aplicación de aspectos de la naturaleza de las ciencias}

Aunque las asignaturas de didáctica de las ciencias experimentales del grado incidieron en diversos aspectos de la naturaleza de las ciencias, desde una perspectiva integrada con los contenidos abordados, conseguir que los futuros maestros superen, en el hacer, algunas ideas ingenuas acerca de la misma es una tarea complicada. Pablo quería introducir el tema de su indagación a partir de un texto motivador, de forma semejante a como suelen hacer los libros de texto. Para ello eligió la historia de la manzana de Newton. Ese texto enfatizaba una visión de la ciencia construida a partir de grandes ideas de genios, en este caso el genio de Newton. Fue discutido cuánto de verdad había en esta versión popular sobre Newton y cómo no encajaba con una visión de ciencias que parte de un problema, punto de partida del trabajo con la metodología de la indagación, remitiéndolo a diferentes materiales en que se abordaban estas cuestiones. Finalmente Pablo consiguió redactar un texto breve, adaptado a los alumnos de primer ciclo, que contemplaba correctamente este aspecto y que, además, servía de punto de partida al problema que finalmente guio su secuencia por indagación. Nuevamente, de no haber surgido la problemática concreta, Pablo no habría conseguido trabajar adecuadamente con la indagación pues su visión de la metodología científica era muy restringida.

\section{La evaluación}

Otro problema al que se enfrentan tanto los maestros en ejercicio como los alumnos de prácticas consiste en la evaluación. ¿Cómo evaluar? ¿Qué herramientas pueden usarse? Aunque han aprendido diversas formas de realizar una evaluación continua, suelen volver a sus propias experiencias personales, estableciendo formas de evaluar que no son consistentes con sus objetivos didácticos. La primera propuesta de Pablo consistió en una evaluación tradicional de primaria: un examen en el que los alumnos completaban espacios, daban definiciones y unían con flechas, prueba que nada tenía que ver con los objetivos que se había planteado. La necesidad de recoger información para dar respuesta a sus preguntas de investigación así como su paulatina comprensión de la metodología de indagación al ir mejorando la propuesta didáctica, le llevó a plantear un esquema de verdadera evaluación continua. Este esquema era coherente con el planteamiento metodológico y con sus objetivos: el trabajo de los alumnos durante la toda la secuencia didáctica era 
efectivamente considerado y la prueba final, que enfrentaba a los niños situaciones nuevas en las que aplicar el conocimiento adquirido, pasó a ser una instancia más de aprendizaje. Es interesante destacar en este caso que una problemática de Pablo era que los niños podían ir mal en esa prueba, pues no estaban acostumbrados. Al quitar el peso de la nota en ella, Pablo se sintió más tranquilo.

"También se puede apreciar que el comportamiento, la profesionalidad (entendida en el caso del alumno como el buen hacer en el aula, las aportaciones en un grupo de trabajo y la atención cuando ésta se requiera) y la constancia quedan reflejadas de una forma porcentualizada que tanto alumnos como padres pueden consultar. A su vez, reflejar en un cuaderno de trabajo los aspectos actitudinales de los alumnos día a día ayudan cuando al maestro se le requieren explicaciones sobre las notas de los pupilos (algo que los padres tienden a hacer cada vez más)" (fuente: Memoria del TFG).

Cabe destacar que la discusión de la evaluación fue un elemento relevante en los tres casos. Los tres estudiantes, en unidades didácticas desarrolladas como trabajos prácticos en asignaturas previas, habían planteado evaluaciones que eran coherentes con sus objetivos y sus planteamientos metodológicos. Sin embargo, al enfrentarse a la práctica, su primera tendencia era evaluar de la forma en que ellos habían sido evaluados y como lo eran los alumnos de los cursos en que estaban, sin darse cuenta de la incoherencia entre metodología y evaluación. Solamente la reflexión y la propia investigación sobre su práctica les permitieron detectar esas incoherencias.

Los mismos temas fueron encontrados también en las otras dos estudiantes aunque en cada caso los planteamientos y soluciones fueron diferentes, dadas las diferencias de contexto, en el que se debe incluir no solamente el contexto escolar específico (escuela, grupo, tema) sino también los conocimientos científicos, visiones sobre la enseñanza de las ciencias, predisposiciones y conocimientos pedagógicos tácitos y sus dificultades particulares para relacionar la teoría con la práctica escolar. En todos los casos, sin embargo, los estudiantes consiguieron implementar con éxito una secuencia didáctica por indagación y experimentar en primera persona los beneficios didácticos de esta metodología:

"A parte de que ahora me siento capaz de ponerme frente a un grupo de alumnos y despertar su interés y su curiosidad por las clases de ciencias, creo que puedo lograr que ellos sientan ilusión por aprender y realmente lleguen a comprender y asimilar los contenidos a través de la experimentación y la observación, eliminando la memorización y la repetición del mapa de la enseñanza de las ciencias" (fuente: Cuestionario final Isabel).

\section{Conclusiones}

El objetivo de este trabajo era estudiar en qué medida el espacio de reflexión construido era útil para auxiliar a los estudiantes de magisterio a dominar mejor la metodología de indagación y a sentirse con confianza para usarla. Los resultados presentados parecen mostrar que la construcción del tercer espacio parece ser una herramienta útil para ayudar a los estudiantes en formación a comprender mejor, durante el diseño, implementación y reflexión de su puesta en práctica, esta metodología. Sin el proceso de reflexión crítica llevado adelante en el tercer espacio, parece difícil que estos alumnos hubieran conseguido implementar la metodología, a pesar de sus intenciones iniciales. Los comentarios de los tres estudiantes, al finalizar sus prácticas, parecen señalar también que la práctica de la metodología reforzó su motivación y confianza para usarla.

Por otra parte, es interesante notar que los temas que aparecieron en ese tercer espacio, y que se constituyeron en problemas para una adecuada implementación de la metodología, correspondieron tanto a aspectos específicos de las mismas como a aspectos generales de la labor docente. Estos aspectos (conducta, evaluación, atención a la diversidad), que vistos con cuidado son 
relevantes para la implementación de esta o cualquier metodología innovadora, no suelen ser tratados con la debida atención en las clases de las didácticas específicas. En el caso presentado, estas cuestiones, que están relacionadas con la acción docente global, independientemente de la enseñanza de las ciencias, se presentaron como serios impedimentos a ser superados para poder implementar la indagación.

Quisiera destacar que la experiencia no solamente parece haber sido rica para los estudiantes sino que lo ha sido para mí. Desde las asignaturas de didáctica de las ciencias experimentales solemos olvidar aspectos esenciales del trabajo diario de los maestros, centrándonos en metodologías y contenidos, y dejando de lado cuestiones como la organización escolar, la conducta, las posibilidades de evaluación, etc., que, sin embargo, parecen ser obstáculos importantes para la implementación de metodologías innovadoras. Así, esta experiencia, además de reforzar mi convicción en la necesidad de establecer más puentes entre la teoría y la práctica en la formación de los docentes para poder mejorar la práctica educativa, me ha permitido repensar estas diversas dificultades, trabajando ahora con los estudiantes las propuestas metodológicas situadas en contextos específicos, con sus problemáticas.

\section{Referencias}

Barnett, R. (1992). Improving Higher Education. Buckingham, SRHE: Open University Press.

Britzman, D. (1986). Cultural myths in the making of a teacher: Biography and social structure in teacher education. Harvard Educational Review, 56, 442-456.

Braun, V. y Clarke, V. (2006) Using thematic analysis in psychology. Qualitative Research in Psychology, 3(2), 77-101.

Bryan, L. A. y Abell, S. K. (1999). The development of professional knowledge in learning to teach elementary science. Journal of Research in Science Teaching, 36, 121-140.

Cañal, P. (2000). El conocimiento profesional sobre las ciencias y la alfabetización científica en primaria. Alambique, 24, 46-56

Cañal de León, P., Criado García-Legaz, A. M., García Carmona, A. y Muñoz, G. (2013). La enseñanza relativa al medio en las aulas españolas de Educación Infantil y Primaria: concepciones didácticas y práctica docente. Investigación en la Escuela, 81, 21-42

Cortés, A.L., de la Gándara, M., Calvo, J.M., Martínez, M.B., Ibarra J., Arlegui, J. y M.J. Gil (2012). Expectativas, necesidades y oportunidades de los maestros en formación ante la enseñanza de las ciencias en la Educación Primaria. Enseñanza de las Ciencias, 30(3), 155-176.

Crawford, B.A. (1999). Is it realistic to expect a preservice teacher to create an inquiry-based classroom? Journal of Science Teacher Education, 10, 175-194.

Creswell, J. W. (1998). Qualitative inquiry and research design: Choosing among five designs. Thousand Oaks, CA: Sage.

Engeström, Y. (1999) Expansive visibilization of work: An activity-theoretical perspective. Computer Supported Cooperative Work, 8, 63-93.

España. Real Decreto 861/2010, de 2 de julio, por el que se modifica el Real Decreto 1393/2007, de 29 de octubre, por el que se establece la ordenación de las enseñanzas universitarias oficiales, Boletín Oficial del Estado, 3 de julio de 2010, núm. 224, pp. 58454-58468.

García Pérez, F. F. ( 2000). Un modelo didáctico alternativo para transformar la educación: el modelo de investigación en la escuela, Scripta Nova. Revista Electrónica de Geografía y Ciencias Sociales, 64.

Gutiérrez, K., Baquedano-López, P., y Tejeda, C. (1999). Rethinking diversity: Hybridity and hybrid language practices in the Third Space. Mind, Culture, \& Activity, 6(4), 286-303. 
Gutiérrez, K., Baquedano-López, P., y Turner, M.G. (1997). Putting language back into the language arts: When the Radical Middle meets the Third Space. Language Arts, 74(5), 368-378.

Haefner, L. A. y Zembal-Saul, C. (2004). Learning by doing? Prospective elementary teachers' developing understandings of scientific inquiry and science teaching and learning. International Journal of Science Education, 26(13), 1653-1674.

Korthagen, F., Loughran, J., Russel, T. (2006). Developing fundamental principles for teacher education programs and practices. Teaching and Teacher Education, 22, 1020-1041.

Minner, D. D., Jurist, A. y Century, J. (2010). Inquiry-based science instruction-what is it and does it matter? Results from a research synthesis years 1984 to 2002. Journal of Research in Science Teaching, 47(4), 474-496

Moje, E. B., Ciechanowski, K. M., Kramer, K. E., Ellis, L. M., Carrillo, R., y Collazo, T. (2004). Working toward third space in content area literacy: An examination of everyday funds of knowledge and discourse. Reading Research Quarterly, 39(1), 38-71

Murphy, C., Neil, P. y Beggs, J. (2007) Primary science teacher confidence revisited: ten years on. Educational Research, 49(4), 415-430

National Research Council (1996). The national science education standards. Washington. DC: The National Academies Press.

National Research Council (2000). Inquiry and the National Science Education Standards. Washington, DC: National Academy Press.

National Research Council (2012). A Framework for K-12 Science Education: Practices, Crosscutting Concepts, and Core Ideas. Washington. DC: The National Academies Press.

Osborne, J. F. y Dillon, J. (2008). Science Education in Europe: Critical reflections. London: Nuffield Foundation.

Porlán, R.; Martín Del Pozo, R.; Rivero, A; Harres, J; Azcárate, P. y Pizzato, M. (2010). El Cambio del Profesorado de Ciencias I: Marco Teórico y Formativo. Enseñanza de las ciencias, 28(1), pp. $31-46$

Porlán, R. y Rivero, A. (1998). El conocimiento de los profesores. Una propuesta formativa en el área de ciencias. Sevilla: Díada.

Rocard, M., Csermely, P., Jorde, D., Lenzen, D., Walwerg-Henriksson, H. y Hemmo, V. (2007). Science Education Now: A Renewed Pedagogy for the Future of Europe. European Commission. Community Research. Recuperado de: http://ec.europa.eu/research/sciencesociety/document_library/pdf_06/report-rocard-onscience-education_en.pdf).

Schwarz, C. (2009). Developing preservice elementary teachers' knowledge and practices through modeling-centered scientific inquiry. Science Education, 93(4), 720-744.

Sykes, G. y Bird, T. (1992). Teacher education and the case idea. Review of Research in Education, 19, 457-521.

Windschitl, M. (2002). Inquiry projects in science teacher education: What can investigative experiences reveal about teacher thinking and eventual classroom practice? Science Education, 87(1), 112-143. 


\section{Sobre la Autora}

Autor: Ileana María Greca Dufranc

Institución: Universidad de Burgos

E-mail:imgreca@ubu.es

Información biográfica: Ileana María Greca Dufranc es investigadora en el área de didáctica de las ciencias experimentales. Sus investigaciones están principalmente relacionadas con la psicología cognitiva y la enseñanza de las ciencias, la enseñanza de tópicos de física moderna, el uso de la historia y la filosofía de las ciencias en la enseñanza de ciencias, el uso de la metodología de la indagación en la escuela y el desarrollo profesional docente de profesores y maestros de ciencias. Su producción bibliográfica incluye 5 libros, 10 capítulos de libros (3 en handbooks internacionales) y 61 artículos de investigación en revistas nacionales e internacionales indexadas, 24 de los cuales en periódicos del ISI Web of Knowledge. Ha participado en 14 proyectos de investigación competitivos. Ha trabajado como docente en todos los niveles educativos y como formadora de docentes e investigadores en la enseñanza de las ciencias. Actualmente es profesora visitante del programa de doctorado en Enseñanza, Historia y Filosofía de las Ciencias de la Universidade Federal da Bahía ( Brasil) y profesora del departamento de Didácticas Especificas de la Universidad de Burgos, donde es docente de varias disciplinas y coordinadora del Grado en Maestro de Educación Primaria.

ORCID: 0000-0003-3674-7985

\section{Investigación en la Escuela}

Revista académica evaluada por pares y de acceso abierto

Número 88

1 de octubre 2016

ISSN 2443-9991

\section{@}

SOMERIIGHISRESERVED Los/as lectores/as pueden copiar, mostrar, y distribuir este artículo, siempre y cuando se de crédito y atribución al autor/es y a Investigación en la Escuela, se distribuya con propósitos no-comerciales, no se altere o transforme el trabajo original. Más detalles de la licencia de Creative Commons se encuentran en http://creativecommons.org/licenses/by-nc-sa/3.0 Cualquier otro uso debe ser aprobado en conjunto por el autor/es, o Investigación en la Escuela.

Contribuya con comentarios y sugerencias en la web de la revista. Por errores y sugerencias contacte a investigacionescuela@,ddcc.uhu.es 


\section{Investigación en la escuela}

Consejo de dirección: Ana Rivero García (Universidad de Sevilla), Nicolás de Alba Fernández (Universidad de Sevilla), Pedro Cañal de León (Universidad de Sevilla), Francisco F. García Pérez (Universidad de Sevilla), Gabriel Travé González, (Universidad de Huelva), Francisco F. Pozuelos Estrada (Universidad de Huelva)

\section{Dirección: Ana Rivero García y Nicolás de Alba Fernández}

Técnico de edición: Francisco Javier López Sánchez

\section{Consejo editorial}

José Félix Angulo Rasco. Universidad de Cádiz Rosa Ma Ávila Ruiz. Universidad de Sevilla Pilar Azcárate Goded. Universidad de Cádiz Juan Bautista Martínez Rodríguez. Universidad de Granada

Nieves Blanco García. Universidad de Málaga Fernando Barragán Medero. Universidad de La Laguna José Carrillo Yáñez. Universidad de Huelva José Contreras Domingo. Universidad de Barcelona. Luis C. Contreras González. Universidad de Huelva Ana $\mathbf{M}^{\mathbf{a}}$ Criado García-Legaz. Universidad de Sevilla Rosario Cubero Pérez. Universidad de Sevilla José $\mathbf{M}^{\mathbf{a}}$ Cuenca López. Universidad de Huelva Jesús Estepa Giménez. Universidad de Huelva Rafael Feito Alonso. Universidad Complutense (Madrid)

Francisco José García Gallardo. Universidad de Huelva

Soledad García Gómez. Universidad de Sevilla J. Eduardo García Díaz. Universidad de Sevilla
Fernando Hernández Hernández. Universidad de Barcelona

Salvador Llinares Ciscar. Universidad de Alicante Alfonso Luque Lozano. Universidad de Sevilla Rosa Martín del Pozo. Universidad Complutense (Madrid)

José Martín Toscano. IES Fernando Herrera (Sevilla) Jaume Martínez Bonafé. Universidad de Valencia F. Javier Merchán Iglesias. Universidad de Sevilla Emilia Moreno Sánchez. Universidad de Huelva. Rosario Ortega Ruiz. Universidad de Córdoba Antonio de Pro Bueno. Universidad de Murcia Fco. de Paula Rodríguez Miranda. Universidad de Huelva

Pedro Sáenz-López Buñuel. Universidad de Huelva Antoni Santisteban Fernández. Universidad Autónoma (Barcelona)

Emilio Solís Ramírez. Catedrático de IES.

$\mathbf{M}^{\mathbf{a}}$ Victoria Sánchez García. Universidad de Sevilla. Magdalena Suárez Ortega. Universidad de Sevilla

\section{Consejo asesor}

Manuel Area Moreira. Universidad de La Laguna

Jaume Carbonell. Director Cuadernos de Pedagogía. Barcelona

César Coll. Universidad de Barcelona

Christopher Day. Universidad de Nothingham. U.K.

Juan Delval. Universidad Nacional de Educación a Distancia

John Elliott. Universidad de East Anglia. Norwich. U.K.

José Gimeno Sacritán. Universidad de Valencia

André Giordan. Universidad de Paris VII y Ginebra

Francisco Imbernón. Universidad de Barcelona

Ángel Pérez Gómez. Universidad de Málaga

Rafael Porlán Ariza. Universidad de Sevilla

Francesco Tonucci. Instituto de Pedagogía del C.N.R. Roma

Jurjo Torres Santomé. Universidad de A Coruña

La revista Investigación en la Escuela desde su origen en 1987 hasta su no 87 (2015) fue editada por Díada Editora. 